\title{
Decreased exposure of simvastatin and simvastatin acid in a rat model of type 2 diabetes
}

\author{
Dan $\mathrm{XU}^{1,2}$, Feng $\mathrm{LI}^{1}$, Mian ZHANG ${ }^{1}$, Ji ZHANG ${ }^{1}$, Can LIU ${ }^{1}$, Meng-yue $\mathrm{HU}^{1}$, Ze-yu ZHONG ${ }^{1}$, Ling-ling JIA ${ }^{1}$, Da-wei WANG ${ }^{2}$, \\ Jie WU², Li LIU ${ }^{1, *}$, Xiao-dong LIU Li * $^{1, *}$ \\ ${ }^{1}$ Key Laboratory of Drug Metabolism and Pharmacokinetics, China Pharmaceutical University, Nanjing 210009, China; ${ }^{2}$ Jiangsu \\ Provincial Institute of Traditional Chinese Medicine, Nanjing 210028, China
}

\begin{abstract}
Aim: Simvastatin is frequently administered to diabetic patients with hypercholesterolemia. The aim of the study was to investigate the pharmacokinetics of simvastatin and its hydrolysate simvastatin acid in a rat model of type 2 diabetes.

Methods: Diabetes was induced in 4-week-old rats by a treatment of high-fat diet combined with streptozotocin. After the rats received a single dose of simvastatin $(20 \mathrm{mg} / \mathrm{kg}$, po, or $2 \mathrm{mg} / \mathrm{kg}$, iv), the plasma concentrations of simvastatin and simvastatin acid were determined. Simvastatin metabolism and cytochrome P4503A (Cyp3a) activity were assessed in hepatic microsomes, and its uptake was studied in freshly isolated hepatocytes. The expression of Cyp3a1, organic anion transporting polypeptide 2 (Oatp2), multidrug resistance-associated protein 2 (Mrp2) and breast cancer resistance protein (Bcrp) in livers was measured using qRT-PCR.

Results: After oral or intravenous administration, the plasma concentrations and areas under concentrations of simvastatin and simvastatin acid were markedly decreased in diabetic rats. Both simvastatin metabolism and Cyp3a activity were markedly increased in hepatocytes of diabetic rats, accompanied by increased expression of hepatic Cyp3a1 mRNA. Furthermore, the uptake of simvastatin by hepatocytes of diabetic rats was markedly increased, which was associated with increased expression of the influx transporter Oatp2, and decreased expression of the efflux transporters Mrp2 and Bcrp.

Conclusion: Diabetes enhances the metabolism of simvastatin and simvastatin acid in rats via up-regulating hepatic Cyp3a activity and expression and increasing hepatic uptake.
\end{abstract}

Keywords: diabetes; hypercholesterolemia; simvastatin; pharmacokinetics; hepatocyte; microsome; Cyp3a; organic anion transporting polypeptide 2; multidrug resistance-associated protein 2; breast cancer resistance protein

Acta Pharmacologica Sinica (2014) 35: 1215-1225; doi: 10.1038/aps.2014.39; published online 25 Aug 2014

\section{Introduction}

Diabetes mellitus (DM) is a well-known risk factor for cardiovascular disease (CVD) and atherosclerotic complications, especially coronary heart disease ${ }^{[1]}$. The combination of diabetes mellitus and CVD history further increases the risk of vascular events ${ }^{[2]}$ and mortality ${ }^{[3]}$. Clinical trials have emphasized the importance of lowering LDL cholesterol and reducing cardiovascular risk in diabetes mellitus ${ }^{[4-6]}$. 3-Hydroxy3-methylglutaryl enzyme A (HMG-CoA) reductase inhibitors (statins) are frequently administered to diabetic patients to reduce cardiovascular risk. Simvastatin, a lipophilic statin, has a high safety profile and is often used for treating hyperlipemia in diabetic patients, although serious symptoms, such

\footnotetext{
* To whom correspondence should be addressed.

E-mail xdliu@cpu.edu.cn (Xiao-dong LIU); liulee@cpu.edu.cn (Li LIU)

Received 2013-12-15 Accepted 2014-03-30
}

as rhabdomyolysis ${ }^{[7]}$, hepatotoxicity ${ }^{[8]}$ and an increased incidence of new-onset diabetes mellitus ${ }^{[9]}$, have been reported. Simvastatin is primarily metabolized in the liver by two separate metabolic pathways. In humans, the oxidative biotransformation of simvastatin is mediated primarily by CYP3A $4^{[10]}$. In the other primary route, the inactive lactone prodrug is hydrolyzed to the pharmacologically active simvastatin acid by carboxylesterases and non-enzymes. The oxidative metabolism of simvastatin acid is catalyzed primarily by CYP3A $4^{[10]}$. Additionally, simvastatin and its hydrolysate simvastatin acid are substrates of organic anion transporting polypeptide 1B1 (OATP1B1/Oatp2) ${ }^{[11-14]}$, an influx transporter expressed on the sinusoidal membrane of hepatocytes. Recent studies have shown that OATP1B1 plays a clinically important role in the hepatic elimination of several drugs including statins, via mediating the hepatic uptake $\mathrm{e}^{[11-15]}$. Both clinical trials and animal experiments have demonstrated that DM may alter the pharmacokinetic behaviors of some drugs via regulating the 
expressions and activities of cytochrome P450s (CYP450s) and drug transporters in the liver ${ }^{[16-22]}$. Breast cancer resistance protein (Bcrp) and multidrug resistance-associated protein 2 (Mrp2) have also been reported to mediate statin transport ${ }^{[11,}$ 23, 24]. All of these results suggest that diabetes modifies the disposition of simvastatin as a result of the alterations of these metabolic enzymes and drug transporters.

The aim of the present study was to investigate the pharmacokinetic behaviors of simvastatin and its hydrolysate simvastatin acid in type 2 diabetic rats induced by a combination of high-fat diet (HFD) and streptozotocin (STZ) injection. The metabolism of simvastatin in hepatic microsomes was measured using in vitro simvastatin depletion. The activity of Cyp3a in hepatic microsomes of diabetic rats was further assessed by the formation of 1-hydroxymidazolam. The mRNA levels of Cyp3a1, Mrp2, Bcrp and Oatp2 in the liver and intestine were measured using quantitative reverse transcriptase polymerase chain reaction (qRT-PCR).

\section{Materials and methods Chemicals}

Simvastatin standard, diazepam and midazolam were obtained from the National Institute for the Control of Pharmaceutical and Biological Products (Beijing, China). Simvastatin acid was purchased from Toronto Research Chemicals Inc (Toronto, ON, Canada). Simvastatin, which was used for pharmacokinetics, was purchased from Dalian Meilun Biotech Corp (Dalian, China). Glucose 6-phosphate, glucose6-phosphate dehydrogenase (Type V), $\beta$-nicotinamide adenine dinucleotide phosphate (NADP) and streptozotocin (STZ) were obtained from Sigma Chemical Co (St Louis, MO, USA). 1-Hydroxymidazolam and Matrigel Matrix were obtained from BD Biosciences (Bedford, MA, USA). All other chemicals used were commercially available and were of analytical grade.

\section{Animals}

Sixty male Sprague-Dawley rats, weighing 100-110 g (aged 4-5 weeks), were obtained from Sino-British Sipper \& BK Lab Animal Ltd (Shanghai, China). The rats were maintained at ambient temperature $\left(23 \pm 1^{\circ} \mathrm{C}\right)$ and relative humidity $(50 \% \pm 10 \%)$ with 12-h light/darkness cycle and were allowed free access to water and food. All of the experimental procedures were approved by the Animal Ethics Committee of China Pharmaceutical University.

\section{Induction of diabetic rats}

The diabetic rats were developed according to our method previously described ${ }^{[19]}$. The rats were randomly divided into three groups: age-matched control (CON) group, high fat diet (HFD) group and diabetes (DM) group. The high-fat diet contained $15 \%$ lard, $5 \%$ sesame oil, $10 \%$ sucrose, $5 \%$ fried peanuts, $2.0 \%$ cholesterol, and $0.2 \%$ cholate. Both the high-fat diet and normal chow were purchased from Jiangsu Xietong Organism Co, Ltd (Nanjing, China). The CON rats were fed normal chow. Both HFD rats and DM rats were fed using a high-fat diet. After 4 weeks of dietary manipulation, the DM rats received an intraperitoneal injection of STZ $(35 \mathrm{mg} / \mathrm{kg}$, dissolved in $\mathrm{pH} 4.5$ citrate buffer $)^{[19]}$. Both HFD and CON rats received only an equivalent volume of citrate buffer. Subsequently, the experimental rats maintained their original diets.

The development of diabetes was confirmed by fasting blood glucose levels using a glucose reagent kit (Jiancheng Biotech Co, Nanjing, China). The animals were considered diabetic only when their fasting blood glucose levels exceeded $16.67 \mathrm{mmol} / \mathrm{L}$ ( $300 \mathrm{mg} / \mathrm{dL}$ ) after 1 week of STZ injection.

On day 14 after STZ or vehicle injection, an oral glucose tolerance test (OGTT) was performed. The experimental rats fasted overnight and received an oral dose of glucose solution $(2 \mathrm{~g} / \mathrm{kg})$. Blood samples were collected via the oculi chorioideae vein under light ether anesthesia at 0 min (just before glucose loading), 15, 30, 60, and 120 min after glucose loading, and serum samples were obtained. The glucose and insulin concentrations in serum were measured using a glucose test kit (Jiancheng Biotech Co, Nanjing, China) and an insulin radioimmunoassay kit (North Institute of Biotech Co, Beijing, China), respectively. Homeostatic model assessment (HOMA) was used to assess the longitudinal changes in insulin resistance (HOMA-IR) ${ }^{[25]}$. The following experiments were performed on the 35th day after STZ injection.

Pharmacokinetics of simvastatin in diabetic rats after oral and intravenous administration

For oral administration, DM rats, CON rats and HFD rats, fasted overnight, and received orally $20 \mathrm{mg} / \mathrm{kg}^{[26,27]}$ of simvastatin (suspended in $0.5 \%$ sodium carboxymethylcellulose). Blood samples $(250 \mu \mathrm{L})$ were collected in heparinized eppendorf tubes via the oculi chorioideae vein at $0.25,0.5,1.0,2.0$, 4.0 , and $8.0 \mathrm{~h}$ after the oral dose under light ether anesthesia. For intravenous administration, $2 \mathrm{mg} / \mathrm{kg}$ of simvastatin (dissolved in mixture of propylene glycol and saline, 1/1) was administered intravenously to DM rats, CON rats and HFD rats via the tail vein. Blood samples $(250 \mu \mathrm{L})$ were collected at $5,10,15,30,45,60,90$, and $120 \mathrm{~min}$ after the intravenous dose under light ether anesthesia. Plasma samples were obtained at $2850 \times \mathrm{g}$ for $5 \mathrm{~min}$ and frozen at $-20^{\circ} \mathrm{C}$ until an analysis was performed. Simvastatin and simvastatin acid concentrations were measured using the LC-MS method.

\section{Preparation of hepatic microsomes and metabolism of simvastatin in hepatic microsomes}

Hepatic microsomes of experimental rats were prepared according to the methods previously described ${ }^{[18,19]}$. The microsomal pellets were re-suspended in phosphate-buffered saline (PBS) ( $\mathrm{pH} 7.4$ ) containing 20\% glycerol and stored at $-80^{\circ} \mathrm{C}$. The protein concentration of the hepatic microsomes was measured using the Bradford method and bovine serum albumin as the standard.

The metabolism of simvastatin in hepatic microsomes was investigated using a simple in vitro substrate depletion approach. Simvastatin $(20 \mu \mathrm{mol} / \mathrm{L})$ was incubated using hepatic microsomes (final level, $0.2 \mathrm{mg} / \mathrm{mL}$ ) in PBS at $37^{\circ} \mathrm{C}$ 
for $5 \mathrm{~min}$. The reaction was initiated by the addition of 40 $\mathrm{mL}$ of NADPH-generating system $(0.5 \mathrm{mmol} / \mathrm{L}$ NADP, 10 mmol/L glucose 6-phosphate, $5 \mathrm{U} / \mathrm{mL}$ glucose-6-phosphate dehydrogenase, and $5 \mathrm{mmol} / \mathrm{L} \mathrm{MgCl}_{2}$ ) (final volume, $200 \mathrm{~mL}$ ). The reaction was terminated by adding $1 \mathrm{~mL}$ of ether acetate at designated incubation times $(0,0.5,1.0,1.5,2,3$, and $5 \mathrm{~min})$. The remaining simvastatin concentrations in the incubation mixtures were determined using the LC-MS method.

It is generally accepted that simvastatin metabolism in the liver is primarily mediated by Cyp3a. The formation of 1-hydroxymidazolam from midazolam, widely used Cyp3a probe, was employed as a marker to assess Cyp3a activity. The incubation mixture (final volume, $200 \mathrm{~mL}$ ) consisted of rat hepatic microsomes (final level, $0.5 \mathrm{mg} / \mathrm{mL}$ ), the NADPHgenerating system, and different midazolam levels in PBS. After a 10 -min preincubation at $37^{\circ} \mathrm{C}$, the reaction was initiated by adding $40 \mathrm{~mL}$ of NADPH-generating system. The final midazolam concentrations in the hepatic incubation mixture were set to be $3.125,6.25,12.5,20,25.0,30.0,50.0$, and 60.0 $\mathrm{mmol} / \mathrm{L}$. All of the above microsomal incubation conditions were in the linear range of reaction rate. The reaction was terminated by adding $200 \mathrm{~mL}$ of acetonitrile after incubation at $37^{\circ} \mathrm{C}$ for $10 \mathrm{~min}$.

\section{Preparation of freshly isolated rat hepatocytes and uptake of simvastatin by hepatocytes}

The experimental rats fasted overnight and were anesthetized using pentobarbital $(60 \mathrm{mg} / \mathrm{kg}$, ip). The rat hepatocytes were isolated using a two-step perfusion method previously described $^{[28]}$. Cell viability was identified using trypan blue test. Hepatocyte suspension was added to pre-coated 24-well plates at a density of $2.5 \times 10^{5}$ cells/well in $500 \mathrm{~mL}$ of Dulbecco's modified Eagle's medium (DMEM). After $4 \mathrm{~h}$ of plating, the cells were used for simvastatin uptake. The incubated cells were washed 3 times using 500 mL of Dulbecco's phosphate buffered saline (DPBS). Subsequently, the cells were incubated with DPBS containing different levels of simvastatin at $37^{\circ} \mathrm{C}$ for $60 \mathrm{~s}$. Simvastatin levels were set to be $10,20,30$, 100,200 , and $500 \mathrm{mmol} / \mathrm{L}$. The uptake was stopped by rinsing the cells 3 times with ice-cold DPBS, and $0.2 \mathrm{~mL}$ of purified water was added to each incubated well. The cells were lysed by 3 freeze-thaw cycles. The simvastatin uptake by the cells was measured using the LC-MS method and expressed as pmol $/ 10^{5}$ cells.

\section{qRT-PCR (quantitative reverse transcriptase polymerase chain} reaction)

qRT-PCR was used to measure mRNA levels of Cyp3a1, Oatp2, Mrp2, and Bcrp in the liver and intestine. The experimental rats were killed under ether anesthesia; their liver and intestine were quickly removed. Total RNA from the frozen tissues was extracted using TRIzol reagent (SunShine Biotechnology, Nanjing, China) according to the manufacturer's instructions. The quality and purity of RNA were determined by UV spectrophotometry at $260 \mathrm{~nm}$ and $280 \mathrm{~nm}$. Quantitative RT-PCR was performed using a cDNA input converted from $2 \mu \mathrm{g}$ of total RNA. Primer sequences of rat mRNA were shown in Table 1. After denaturing at $95^{\circ} \mathrm{C}$ for $2 \mathrm{~min}$, the amplification was obtained by 40 cycles of $95^{\circ} \mathrm{C}$ for $5 \mathrm{~s}$ and $60^{\circ} \mathrm{C}$ for $30 \mathrm{~s}$. Melting curves were performed to investigate the specificity of the PCR reaction. For normalization of the gene levels, $\beta$-actin was used to correct minor variations in the input RNA amount or inefficiencies of the reverse transcription. The relative quantification (RQ) of each gene expression was calculated according to comparative $\mathrm{Ct}$ method using this formula: $\mathrm{RQ}=2^{-\Delta \mathrm{Ct}}$.

Table 1. Primer sequences of rat mRNA.

\begin{tabular}{|c|c|}
\hline Gene & Sequences \\
\hline \multirow[t]{2}{*}{ Сур3а1 } & Forward 5'-GAAACCCGCCTGGATTCTGTG-3' \\
\hline & Reverse 5'-CCATATCGGATAGGGCTGTATGAG-3' \\
\hline \multirow[t]{2}{*}{ Oatp2 } & Forward 5'-CСАCTGCTGAGGTAGTCTTCCTTATG-3' \\
\hline & Reverse 5'-CAGAGCCAGAATGCTAAGTGTGC-3' \\
\hline \multirow[t]{2}{*}{ Mrp2 } & Forward 5'-CACGGTCATCACCATCGCTCAC-3' \\
\hline & Reverse 5'-AGTTCTTCAGGACTGCCATACTCG-3' \\
\hline \multirow[t]{2}{*}{ Bcrp } & Forward 5'-GTACTTTGCATCAGCAGGTTACCACT-3' \\
\hline & Reverse 5'-ATTACAGCCGAAGAATCTCCGTTG-3' \\
\hline \multirow[t]{2}{*}{$\beta$-Actin } & Forward 5'-CAGTCGGTTGGAGCGAGCAT-3' \\
\hline & Reverse 5'-GGACTTCCTGTAACAACGCATCT-3' \\
\hline
\end{tabular}

\section{Drug assays}

Simvastatin and simvastatin acid levels in the biological samples were determined using LC-MS as previously described with minor modification ${ }^{[29]}$. The diclofenac sodium solution (internal standard, final concentration, $1 \mathrm{mg} / \mathrm{mL}$ ) of $10 \mathrm{~mL}$ and ether acetate of $1 \mathrm{~mL}$ were added to $100 \mathrm{~mL}$ of the biological sample. After shaking for $10 \mathrm{~min}$, the mixture was subsequently centrifuged at $1820 \times g$ for $10 \mathrm{~min}$. The organic phase was first transferred into clean tubes, then dried under reduced pressure using an integrated SpeedVac SPD2010-230 system (Thermo Fisher Scientific, Waltham, MA, USA). The residue was reconstituted in $100 \mathrm{~mL}$ of methanol and centrifuged at $45570 \times g$ for $10 \mathrm{~min}$. Subsequently, the $5 \mathrm{~mL}$ supernatant was injected into the LC-MS system.

The assay was performed using the LC/MS 2010A system (Shimadzu, Kyoto, Japan). Liquid chromatographic separations were achieved using a Waters Symmetry C18 column $(150 \mathrm{~mm} \times 2.1 \mathrm{~mm}$, internal diameter $5.0 \mu \mathrm{m})$. The column and autosampler tray temperatures were kept constant at $40^{\circ} \mathrm{C}$ and $4^{\circ} \mathrm{C}$, respectively. The mobile phase consisted of water (containing $0.02 \%$ formic acid and $12 \mu \mathrm{mol} / \mathrm{L}$ sodium acetate) (A) and methanol (B) $(17: 83, v / v)$, which was delivered at ate of $0.2 \mathrm{~mL} / \mathrm{min}$. The samples were ionized using positive-ion electrospray ionization (ESI) probe in the positive-ion mode under the following source conditions: gas flow, $4.5 \mathrm{~L} / \mathrm{min}$; curve dissolution line (CDL) voltage was fixed as in tuning, CDL temperature, $250{ }^{\circ} \mathrm{C}$; and block temperature, $400^{\circ} \mathrm{C}$. Mass spectra were obtained at a dwell time of $0.2 \mathrm{~s}$ and $1 \mathrm{~s}$ for 
selected ion monitoring (SIM) and scan mode accordingly. A SIM analysis was performed as follows: for simvastatin, $[\mathrm{M}+\mathrm{Na}]^{+}, m / z=441$; for simvastatin acid, $[\mathrm{M}+\mathrm{Na}]^{+}, m / z=459$; and for internal standard, $[\mathrm{M}+\mathrm{H}]^{+}, m / z=296$. Peak areas for all of the components were automatically integrated using LC/MS solution Version 2.04 (Shimadzu). The method was validated throughout the concentration range of 37.5-500.0 $\mathrm{ng} / \mathrm{mL}$ for simvastatin and $39.0-5000.0 \mathrm{ng} / \mathrm{mL}$ for simvastatin acid with correlation coefficients of 0.999 for simvastatin and 0.999 for simvastatin acid.

In the incubation mixture, 1-hydroxymidazolam level was measured using HPLC. The incubation samples were centrifuged twice for $10 \mathrm{~min}$. Subsequently, the supernatant (20 $\mathrm{mL}$ ) was applied to the HPLC system. The HPLC system was composed of an LC-10AD pump (Shimadzu), a model SPD10A UV absorbance detector (Shimadzu) set at $220 \mathrm{~nm}$, and a Symmetry C18 $(150 \mathrm{~mm} \times 4.6 \mathrm{~mm}$, internal diameter $5 \mu \mathrm{m})$ (Waters). The mobile phase consisted of water (containing 10 $\mathrm{mmol} / \mathrm{L}$ ammonium acetate) (A) and acetonitrile (B) $(60 / 40$, $v / v)$; the flow rate was set at $1.0 \mathrm{~mL} / \mathrm{min}$. The linear range in the incubation mixture was $0.10-1.00 \mu \mathrm{g} / \mathrm{mL}$.

\section{Pharmacokinetic analysis}

The concentration-time data of simvastatin and simvastatin acid for each rat was individually analyzed using noncompartmental analysis and Phenix WinNonlin 6.1 (Pharsight, St Louis, MO, USA). The area under the plasma concentrationtime profile $\left(\mathrm{AUC}_{0-\infty}\right)$ was calculated using the trapezoidal rule, with extrapolation to infinity. The peak concentration $\left(C_{\max }\right)$ and the time to reach $C_{\max }$ were obtained directly from the plasma concentration-time profiles. The terminal elimination constant $(K)$ was obtained from the least-squares linear regression slope of the natural logarithm of concentration versus time, and the terminal elimination half-life $\left(T_{1 / 2}\right)$ was calculated as $0.693 / K$. The mean residence time (MRT) was calculated as the area under the first moment curve divided by $\mathrm{AUC}_{0-\infty}$. For intravenous administration, the clearance $(\mathrm{Cl})$ was calculated as dose $/ \mathrm{AUC}_{0-\infty}$.

In the hepatic microsomal incubation, the area under the simvastatin concentration-time curve $\left(\mathrm{AUC}_{0-\mathrm{t}}\right)$ was calculated using the linear trapezoidal rule. The clearance of simvastatin in hepatic microsomes $\left(\mathrm{Cl}_{\text {apph }}\right)$ was estimated as the initial dose (final volumexinitial levels of simvastatin) divided by $\mathrm{AUC}_{0-\mathrm{t}}$.

The Michaelis-Menten equation

$$
V=\frac{V_{\max }+[\mathrm{S}]}{K_{\mathrm{m}}+[\mathrm{S}]}
$$

and Hill equation

$$
V=\frac{V_{\max }+[\mathrm{S}]^{\gamma}}{\mathrm{S}_{50}+[\mathrm{S}]^{\gamma}}
$$

were used to characterize the kinetics of 1-hydroxymidazolam formation from midazolam in rat hepatic microsomes, where $\mathrm{V}, \mathrm{S}, K_{\mathrm{m}}$ and $V_{\max }$ were the velocity of the reaction, the substrate concentration, the Michaelis-Menten constant and the maximum velocity, respectively. $\mathrm{S}_{50}$ was the substrate concen- tration showing the half- $V_{\max }$ and $\mathrm{g}$ was the Hill coefficient. For Michaelis-Menten kinetics, intrinsic clearance $\left(\mathrm{Cl}_{\text {int }}\right)$ was calculated as $V_{\max } / K_{\mathrm{m}}$ and for sigmoidal kinetics, maximum clearance $\left(\mathrm{Cl}_{\max }\right)^{[30]}$ was calculated as

$$
\mathrm{Cl}_{\max }=\frac{V_{\max } \times(\gamma-1)}{\mathrm{S}_{50} \times \gamma^{\times}(\gamma-1)^{1 / \gamma}}
$$

In the present study, if the Hill coefficient was more than 1.2 , the kinetic data were fit to the Hill equation.

\section{Statistical analysis}

The data are presented as the mean \pm standard deviation (SD) values. Significant differences between groups were evaluated using the one-way analysis of variance. If the analysis results were significant, the differences between groups were estimated using the Student-Newman-Keuls multiple-comparison test for post hoc analysis. A $P$ value of less than 0.05 was considered statistically significant.

\section{Results}

\section{Establishment of the diabetic rat model}

The biochemical parameters were measured in the plasma of CON rats, HFD rats and DM rats on $\mathrm{d} 7$ after the administration of STZ (Table 2). Markedly elevated levels of glucose, triglyceride, and total cholesterol were found in the serum samples of DM rats. DM rats also developed diabetic symptoms, such as polyphagia, polyuria and polydipsia, accompanied by significantly increased HOMA-IR values. Compared with CON rats, HFD rats showed higher triglyceride, total cholesterol and lower insulin concentrations. The OGTT results showed that DM rats had significantly higher serum glucose and lower insulin concentrations induced by glucose loading than CON rats and HFD rats, resulting in significant increases in AUC values of glucose and decreases in AUC of insulin (Figure 1). These indexes were similar to the physiopathological state of type 2 diabetes, indicating that the DM rats may be considered as having type 2 diabetes ${ }^{[31,32]}$. Insulin resistance also occurred in HFD rats, in which the insulin and glucose levels induced by glucose loading were higher than those in CON rats (Figure 1 ).

\section{Pharmacokinetics of simvastatin in DM rats after oral and intravenous administration of simvastatin}

The plasma concentrations of simvastatin and its hydrolysate simvastatin acid were measured in CON, DM and HFD rats after oral administration of simvastatin $(20 \mathrm{mg} / \mathrm{kg})$ to experimental rats (Figure 2A and 2B). The corresponding pharmacokinetic parameters were estimated (Table 3). Compared with $\mathrm{CON}$ rats, DM rats had lower plasma concentrations of simvastatin and simvastatin acid, which resulted in lower exposure $\left(C_{\max }\right.$ and $\left.\mathrm{AUC}_{0-\infty}\right)$. The mean $\mathrm{AUC}_{0-\infty}$ and $C_{\max }$ in DM rats were $72 \%$ for simvastatin in CON rats and $56 \%$ for simvastatin acid. Compared with CON rats, HFD rats showed lower concentrations and AUC values in plasma concentrations of simvastatin and simvastatin acid.

To investigate whether the decreased oral exposure of 
Table 2. Biochemical parameters in experimental rats on d 7 following STZ.

\begin{tabular}{|c|c|c|c|}
\hline Parameters & CON & HFD & DM \\
\hline Initial body weight (g) & $132.4 \pm 6.85$ & $132.3 \pm 7.6$ & $138.3 \pm 4.51$ \\
\hline Final body weight (g) & $330.8 \pm 11.2$ & $310.6 \pm 12.0$ & $317.8 \pm 18.0$ \\
\hline Serum glucose (mmol/L) & $5.92 \pm 0.40$ & $6.09 \pm 0.78$ & $29.92 \pm 2.02^{\mathrm{cf}}$ \\
\hline Serum triglyceride (mmol/L) & $1.68 \pm 0.25$ & $2.12 \pm 0.23^{c}$ & $2.57 \pm 0.47^{\mathrm{ce}}$ \\
\hline Serum total cholesterol $(\mathrm{mmol} / \mathrm{L})$ & $1.91 \pm 0.29$ & $2.84 \pm 0.55^{c}$ & $4.99 \pm 1.17^{\mathrm{cf}}$ \\
\hline
\end{tabular}

Data were expressed as mean \pm SD of 6 rats, ${ }^{b} P<0.05,{ }^{c} P<0.01$ vs CON rats. ${ }^{e} P<0.05,{ }^{f} P<0.01$ vs HFD rats.

Table 3. Pharmacokinetic parameters of simvastatin and simvastatin acid after oral $(20 \mathrm{mg} / \mathrm{kg})$ and intravenous $(2 \mathrm{mg} / \mathrm{kg})$ administration of simvastatin to DM, HFD, and CON rats.

\begin{tabular}{|c|c|c|c|}
\hline Parameters & $\mathrm{CON}$ & HFD & DM \\
\hline \multicolumn{4}{|l|}{ Oral administration } \\
\hline$T_{\max }(\mathrm{h})$ & $1.38 \pm 0.63$ & $0.75 \pm 0.84$ & $0.75 \pm 0.25$ \\
\hline$C_{\max }(\mathrm{ng} / \mathrm{mL})$ & $252.50 \pm 29.91$ & $197.28 \pm 65.64$ & $181.31 \pm 14.14^{\circ}$ \\
\hline $\mathrm{AUC}_{0-\infty}\left(\mathrm{ng} \cdot \mathrm{h} \cdot \mathrm{mL}^{-1}\right)$ & $1399.40 \pm 143.81$ & $820.01 \pm 72.45^{c}$ & $1003.79 \pm 40.92^{\mathrm{cf}}$ \\
\hline \multicolumn{4}{|l|}{ Simvastatin acid } \\
\hline$T_{\max }(\mathrm{h})$ & $1.06 \pm 0.72$ & $1.25 \pm 0.29$ & $0.95 \pm 0.37$ \\
\hline$C_{\max }(\mathrm{ng} / \mathrm{mL})$ & $301.75 \pm 64.33$ & $147.63 \pm 10.41^{\mathrm{c}}$ & $167.70 \pm 40.59^{c}$ \\
\hline $\mathrm{AUC}_{0-\infty}\left(\right.$ ng.h.mL $\left.L^{-1}\right)$ & $1312.86 \pm 210.40$ & $727.47 \pm 49.27^{c}$ & $744.10 \pm 91.45^{c}$ \\
\hline \multicolumn{4}{|c|}{ Simvastatin } \\
\hline $\mathrm{AUC}_{120}\left(\mathrm{ng} \cdot \mathrm{h} \cdot \mathrm{mL}^{-1}\right)$ & $388.43 \pm 36.35$ & $507.57 \pm 63.81$ & $289.72 \pm 33.87^{\text {cf }}$ \\
\hline$A \cup C_{0-\infty}\left(\right.$ ng $\left.h \cdot m^{-1}\right)$ & $466.27 \pm 75.18$ & $642.07 \pm 130.08$ & $369.12 \pm 49.45^{f}$ \\
\hline$T_{1 / 2}(\min )$ & $42.45 \pm 9.17$ & $52.96 \pm 21.48$ & $47.38 \pm 12.20$ \\
\hline $\mathrm{Cl}\left(\mathrm{L} \cdot \mathrm{h}^{-1} \cdot \mathrm{kg}^{-1}\right)$ & $4.36 \pm 0.61$ & $3.21 \pm 0.64^{b}$ & $5.49 \pm 0.72^{f}$ \\
\hline MRT (min) & $66.42 \pm 12.8$ & $80.88 \pm 20.20$ & $73.18 \pm 22.72$ \\
\hline \multicolumn{4}{|l|}{ Simvastatin acid } \\
\hline $\mathrm{AUC}_{120}\left(\mathrm{ng} \cdot \mathrm{h} \cdot \mathrm{mL}^{-1}\right)$ & $843.17 \pm 235.83$ & $1225.17 \pm 316.67$ & $486.33 \pm 53.50^{\text {bf }}$ \\
\hline$A \cup C_{0-\infty}\left(n g \cdot h \cdot m L^{-1}\right)$ & $946.33 \pm 300.33$ & $1294.00 \pm 329.67$ & $492.33 \pm 53.83^{b f}$ \\
\hline$T_{1 / 2}(\min )$ & $31.55 \pm 9.78$ & $29.90 \pm 3.98$ & $20.39 \pm 3.82^{b f}$ \\
\hline
\end{tabular}

Data were expressed as mean \pm SD of 6 rats, ${ }^{b} P<0.05,{ }^{c} P<0.01$ vs CON rats. ${ }^{f} P<0.01$ vs HFD rats.

simvastatin and simvastatin acid in the plasma of DM rats resulted from enhanced systemic clearance, the pharmacokinetic behaviors of simvastatin and simvastatin acid (Figure $2 \mathrm{C}$ and 2D) in experimental rats were studied after an intravenous dose of simvastatin $(2 \mathrm{mg} / \mathrm{kg})$. Consistent with our expectation, DM rats showed lower plasma concentrations of simvastatin and simvastatin acid, accompanied by lower
$\mathrm{AUC}_{120}$ value (only $74 \%$ for simvastatin in CON rats and $58 \%$ for simvastatin acid). In contrast to the oral administration of simvastatin in DM rats, the plasma concentrations and exposures of both simvastatin and simvastatin acid in HFD rats were increased rather than decreased compared with CON rats. 

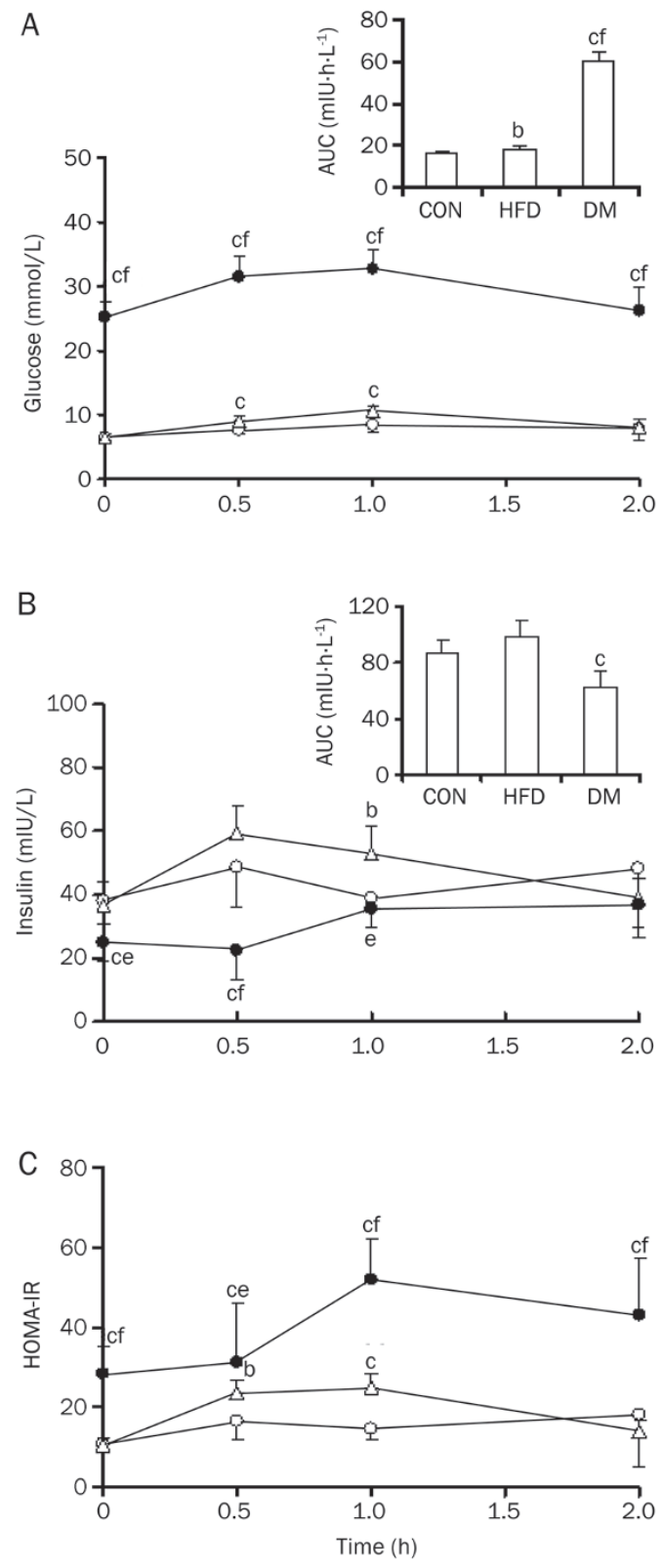

Figure 1. Serum glucose (A), insulin (B) concentrations and HOMA-IR (C) in DM (solid cycle), HFD (open triangle), and CON (open cycle) rats after oral glucose loading. Data were expressed as mean $\pm S D$ of 6 rats, ${ }^{\mathrm{b}} P<0.05,{ }^{\mathrm{c} P}<0.01$ vs CON rats. ${ }^{\mathrm{e}} P<0.05,{ }^{\mathrm{f}} P<0.01$ vs HFD rats.

\section{Simvastatin metabolism in hepatic microsomes and СурЗа activity}

Simvastatin is primarily eliminated in the liver via metabolism. To investigate whether the alteration in the pharmacokinetics of simvastatin was derived from the enhancement of simvastatin metabolism in the liver of DM rats, the metabolism of simvastatin in hepatic microsomes of CON, HFD and DM rats was measured (Figure $3 \mathrm{~A}$ ) using a simple in vitro substrate depletion, and clearances in hepatic microsomes of CON, HFD and DM rats were estimated (Table 4). The results demonstrated that simvastatin metabolism was markedly
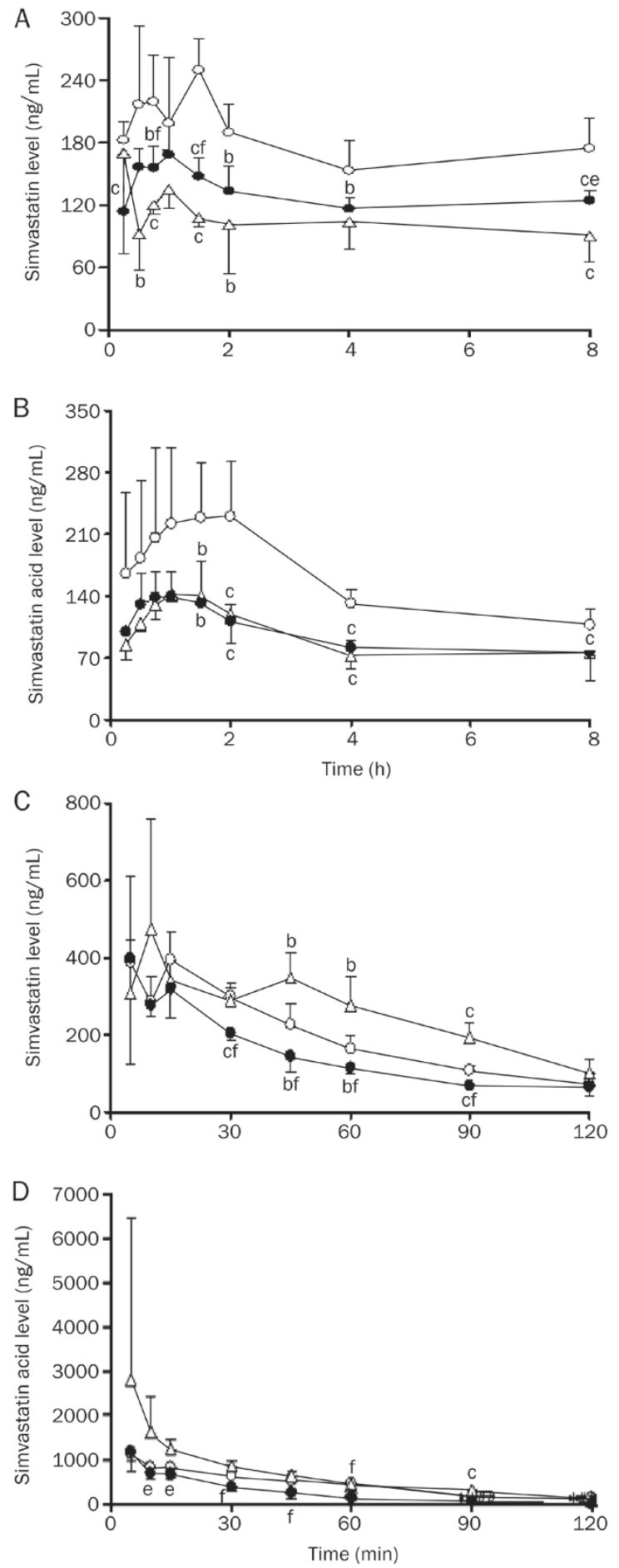

Figure 2. Simvastatin (A, C) and simvastatin acid (B, D) concentrations in plasma of DM (solid cycle), HFD (open triangle), and CON (open cycle) rats after an oral dose (20 mg/kg, A, B) and an intravenous dose (2 mg/kg, B, C) of simvastatin to experimental rats. Data were expressed as mean $\pm S D$ of $4-5$ rats. ${ }^{b} P<0.05,{ }^{c} P<0.01$ vs CON rats. ${ }^{e} P<0.05,{ }^{f} P<0.01$ vs HFD rats.

$(P<0.05)$ enhanced in hepatic microsomes of DM rats, leading to a significant $(P<0.05)$ increase in simvastatin clearance $(1.22-$ fold of CON rats). The HFD rats also showed a trend toward 
A

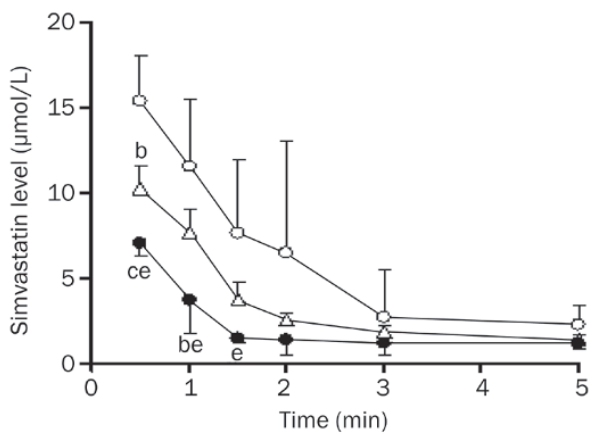

C

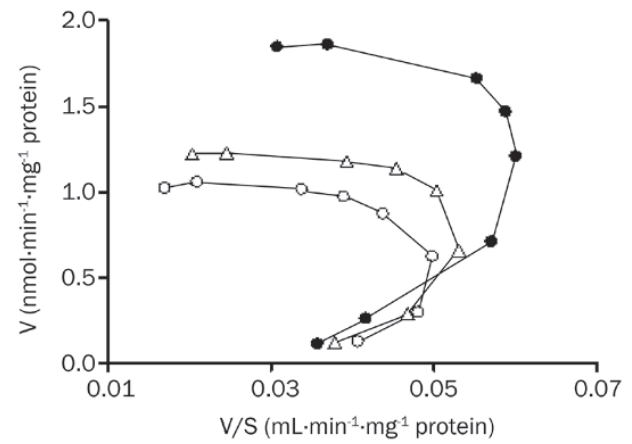

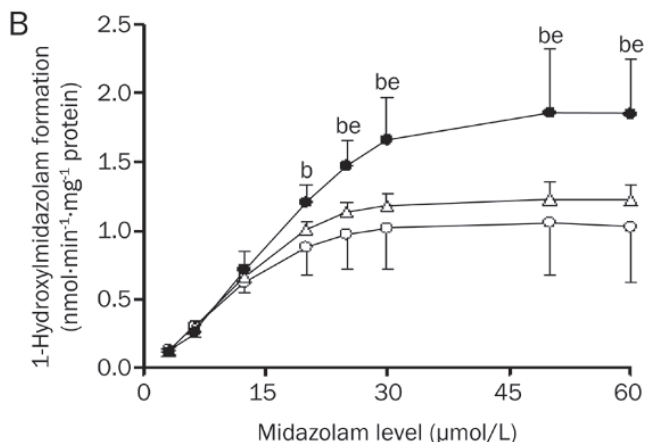

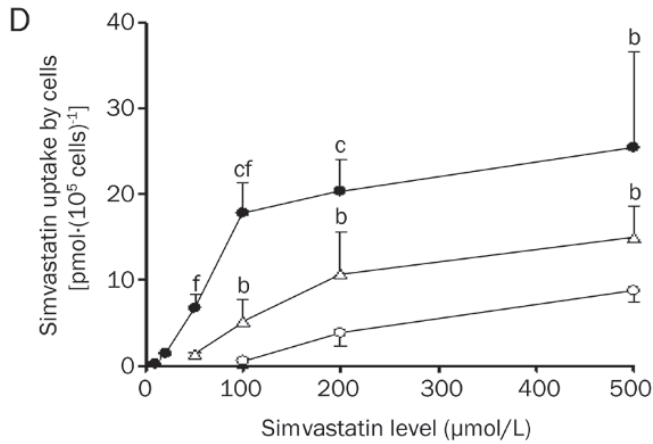

Figure 3. Simvastatin depletion in rat hepatic microsomes (A), 1-hydroxymidazolam formation from midazolam in rat hepatic microsomes (B) and the corresponding Eadie-Hofstee plots (C), and uptake of simvastatin (D) by hepatocytes of CON rats (open cycle), HFD rats (open triangle) and DM rats (solid cycle). Data were expressed as mean \pm SD of $3-4$ rats. ${ }^{b} P<0.05,{ }^{\circ} P<0.01$ vs CON rats. ${ }^{e} P<0.05,{ }^{f} P<0.01$ vs HFD rats.

Table 4. Kinetic parameters of midazolam and simvastatin metabolism in hepatic microsomes of CON, HFD, and DM rats.

\begin{tabular}{|c|c|c|c|}
\hline Parameters & CON & HFD & DM \\
\hline \multicolumn{4}{|l|}{ 1-Hydroxymidazolam formation } \\
\hline y & $2.29 \pm 0.25$ & $2.30 \pm 0.26$ & $2.07 \pm 0.24$ \\
\hline$V_{\max }\left(\mathrm{nmol} \cdot \mathrm{min}^{-1} \cdot \mathrm{mg}^{-1}\right.$ protein $)$ & $1.09 \pm 0.40$ & $1.28 \pm 0.14^{\mathrm{b}}$ & $1.83 \pm 0.41^{\text {be }}$ \\
\hline $\mathrm{S}_{50}(\mu \mathrm{mol} / \mathrm{L})$ & $9.70 \pm 3.61$ & $11.35 \pm 0.99$ & $13.73 \pm 2.34$ \\
\hline $\mathrm{Cl}\left(\mu \mathrm{L} \cdot \mathrm{min}^{-1} \cdot \mathrm{mg}^{-1}\right.$ protein $)$ & $56.66 \pm 6.44$ & $57.19 \pm 5.08$ & $66.56 \pm 3.58^{\text {be }}$ \\
\hline \multicolumn{4}{|l|}{ Simvastatin depletion } \\
\hline $\mathrm{AUC}_{0-5}\left(\mu \mathrm{moL} \cdot \min \cdot \mathrm{L}^{-1}\right)$ & $32.34 \pm 14.91$ & $20.95 \pm 2.04$ & $13.17 \pm 3.35^{b}$ \\
\hline $\mathrm{Cl}\left(\mathrm{mL} \cdot \mathrm{min}^{-1} \cdot \mathrm{mg}^{-1}\right.$ protein $)$ & $3.56 \pm 1.57$ & $4.80 \pm 0.45$ & $7.89 \pm 1.77^{\mathrm{b}}$ \\
\hline
\end{tabular}

Data were expressed as mean \pm SD of $3-4$ rats. ${ }^{b} P<0.05$ vs CON rats. ${ }^{e} P<0.05$ vs HFD rats.

increasing clearance in hepatic microsomes; however, no significance was obtained.

Simvastatin metabolism in the liver is primarily mediated by Cyp3a. Cyp3a activities in the hepatic microsomes of experimental rats were documented using formation of 1-hydroxymidazolam (Figure 3B). Similar to simvastatin metabolism, formation rates of 1-hydroxymidazolam were markedly enhanced in hepatic microsomes of DM rats. Additional studies showed that data on the formation rates of 1-hydroxymidazolam in hepatic microsomes of experimental rats were not fitted using the simple Michaelis-Menten equation; however, good fittings were obtained using the Hill equation. Corresponding kinetic parameters were estimated (Table 4). A significant decrease in $\mathrm{AUC}_{0-5}(40 \%$ of $\mathrm{CON}$ rats) and increased clearance (2.22-fold of CON rats) of midazolam were found in hepatic microsomes of DM rats, implying enhanced hepatic Cyp3a activity.

\section{Uptake of simvastatin by hepatocytes}

Uptake of statins by hepatocytes is considered a ratedetermining process in the overall hepatic elimination of statins $^{[33,34]}$. The uptake of simvastatin by hepatocytes was investigated using freshly isolated rat hepatocytes from experimental rats (Figure 3D). The results showed that the uptake 
of simvastatin by hepatocytes of DM rats was markedly enhanced.

\section{qRT-PCR}

Several efflux transporters (Bcrp and Mrp2) and influx transporter (Oatp2) were involved in statin transport. The mRNA levels of Cyp3a1 and these transporters in the liver and intestines were measured using qRT-PCR (Figure 4). Compared with the intestines, the liver expressed higher levels of Bcrp, Mrp2, Oatp2, and Cyp3a1 mRNA. The intestinal Oatp 2 mRNA (relative to $\beta$-actin mRNA) levels were less than 0.0001 , indicating a minor contribution to intestinal drug absorption. Diabetes significantly enhanced the expression of hepatic Cyp3a1 mRNA, inducing an increase by 2.7-fold in $\mathrm{CON}$ rats. An increase in the expression of hepatic Oatp2 was also observed in DM rats (increase by $20 \%$ in CON rats and $50 \%$ in FHD rats, respectively), although no significance was obtained. By contrast, significant decreases in hepatic Bcrp and Mrp2 mRNA occurred in the liver of DM rats. DM altered mRNA levels of Cyp3a1, Bcrp, and Mrp2 in the intestines; no significance was obtained because of the large individual differences.
A

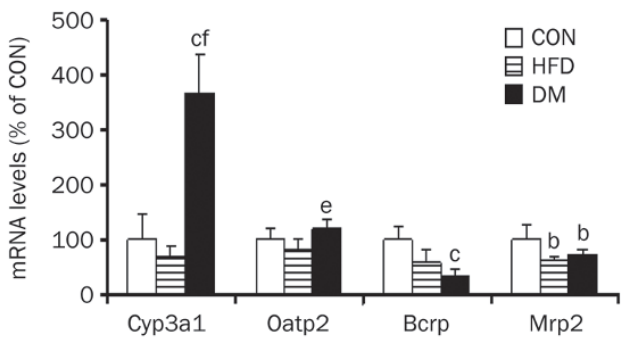

B

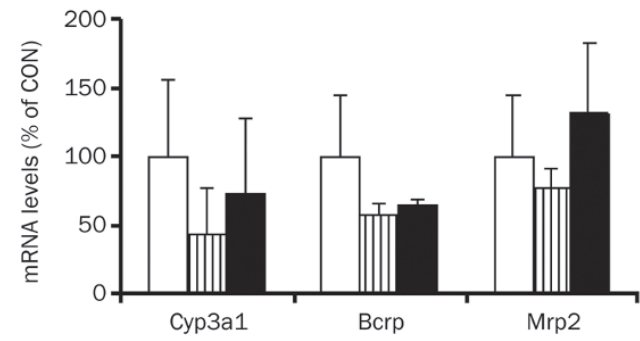

Figure 4. mRNA levels of transporters and Cyp3a1 in liver (A) and intestine (B) of CON, HFD, and DM rats. Data were expressed as mean \pm SD of 4 rats. ${ }^{\mathrm{b}} P<0.05,{ }^{\mathrm{c}} P<0.01$ vs $\mathrm{CON}$ rats. ${ }^{\mathrm{e}} \mathrm{P}<0.05,{ }^{\mathrm{f}} P<0.01$ vs HFD rats.

\section{Discussion}

Accumulating reports have shown that diabetes alters the metabolism rates of drugs via affecting activities and expressions of CYP450s and drug transporter in the liver ${ }^{[15,17,20,35] .}$ Simvastatin, a substrate of Cyp3 and Oatp2/OATP1B1, is often used for improving hypercholesterolemia associated with DM. The aim of the present study was to investigate the effect of type 2 diabetes on simvastatin pharmacokinetics. Type 2 diabetic rats were developed using the combination of an HFD and a low dose of STZ. The results showed that the developed diabetic rats had higher levels of glucose in the serum, accompanied by increased HOMA-IR. Additionally, the rats showed abnormalities in lipid metabolism as evidenced by high triglyceride and cholesterol levels. All of the syndromes were similar to those in type 2 diabetes patients, which indicated that the developed type 2 diabetic rats may reflect the natural history and metabolic characteristics of human type 2 diabetes. The primary finding of this study was that diabetes significantly decreased concentrations in plasma, accompanied by lower exposure $\left(C_{\max }\right.$ and AUC) of simvastatin and its hydrolysate simvastatin acid (Figure 2) after oral administration. Using intravenous administration, we verified that the decreased exposure of simvastatin and simvastatin acid was derived from enhanced systematic clearance. It is generally accepted that simvastatin and simvastatin acid in the liver are metabolized via Cyp3a, and simvastatin clearance in hepatic microsomes was therefore measured using in vitro simvastatin depletion. The results were consistent with findings in vivo that clearance of simvastatin in hepatic microsomes of DM rats was significantly increased. The activity and expression of Cyp3a in the liver of experimental rats were determined using 1-hydroxymidazolam formation from midazolam, a Cyp3a probe, and qRT-PCR. The results clearly demonstrated marked enhancement of the activity in hepatic microsomes of DM rats, which was consistent with the increase in simvastatin metabolism. Similarly, a significant increase in the levels of Cyp3a1 mRNA was observed in the liver of DM rats. We previously reported significant enhancement of Cyp3a in hepatic microsomes of DM rats using norverapamil formation from verapamil, another type of Cyp3a probe ${ }^{[18,19]}$. All of these results suggested that the enhanced activity and expression of hepatic Cyp3a were the main contributors to the increases in systemic clearance of simvastatin and simvastatin acid.

The uptake of drugs by hepatocytes is also considered a rate-determining process in the hepatic clearance of statins via OATPs $^{[33,34]}$. The uptake of simvastatin by hepatocytes and the expression of Oatp2 were also investigated. Significantly enhanced uptake of simvastatin by hepatocytes was observed in DM rats, accompanied by a trend toward increasing the expression of hepatic Oatp2 mRNA. Simvastatin was also reported to be a substrate of Mrp2 and Bcrp ${ }^{[11,22,23]}$, implying that the two efflux transporters may be involved in the elimination of simvastatin via the bile. However, a report showed that only a small percentage of simvastatin is eliminated via feces in the form of simvastatin or simvastatin acid ${ }^{[36]}$. Our preliminary results showed that the excreted amount of simvastatin as simvastatin and simvastatin acid via the bile and feces was less than $1 \%$ of the administered dose. Furthermore, the expression of Mrp2 and Bcrp in the liver was downregulated. All of these findings excluded the contribution of the two efflux transporters to promoting the elimination of simvastatin and simvastatin acids in the liver of DM rats. Although intestinal Cyp3a may be involved in the presystem- 
atic metabolism of simvastatin after an oral dose, the intestinal Cyp3a1 mRNA levels were down-regulated under diabetic conditions. Our previous study demonstrated the suppression of Cyp3a activity and the expression in the intestines of DM rats ${ }^{[18]}$. No significant alterations in the expressions of Bcrp mRNA and Mrp2 mRNA were observed in the intestines of DM rats. Therefore, the findings in the intestines of DM rats did not explain the decreased exposure of simvastatin and simvastatin acid after an oral dose of simvastatin. All the results may support the conclusion that the up-regulation of both Cyp3a activity and expression as well as the enhanced hepatic uptake contributed, at least in part, to the increase in the hepatic metabolism of simvastatin and simvastatin acid under diabetic conditions, thus leading to lower exposures of simvastatin and simvastatin acid.

The mechanism underlying the elevations in hepatic Сyp3a and Oatp2 expression in DM rats has not been established. The present study showed that marked accumulations of serum cholesterol, triglyceride, and insulin resistance occurred in both HFD and DM rats. The activity and expression of hepatic Cyp3a in DM rats were associated with HOMA-IR, which suggests that insulin resistance or relative insufficiency of insulin may explain the affecting expression and activity of hepatic Cyp3a. Several reports have demonstrated that insulin and ketone bodies participating in the regulation of cytochrome P450 isozymes in the liver of DM rats and insulin treatment reversed or partially reversed alteration induced by diabetes $^{[17,37-39]}$. The elevated total serum cholesterol and triglyceride levels in DM rats were consistent with the increases in activity and expression of hepatic Cyp3a, suggesting that high cholesterol and triglyceride levels may explain inducing Cyp3a expression and activity. In agreement with this deduction, Irizar et al reported that the administration of cholesterol-rich diets enhanced Cyp3a levels in atherosclerotic rabbits ${ }^{[40]}$. Lucas et al found that a positive correlation existed between CYP2E1 activity and serum cholesterol or triglycerides in diabetes patients ${ }^{[41]}$. Similarly, Watson et al found that $16 \beta$-testosterone hydroxylation in hepatic microsomes of $o b / o b$ mice was higher than that in lean mice ${ }^{[42]}$. However, the above-mentioned deduction did not explain the findings in HFD rats. Although the elevated total serum cholesterol and triglyceride in HFD rats were also observed, the activity and expression of hepatic Cyp3a were minimally affected.

The higher activity of Cyp3a in DM rats was also accompanied by a higher glucose level. However, Stewart et al found that glucose consumption in rats may decrease the activity of Cyp2c11 and Cyp3a using testosterone as a probe agent ${ }^{[43]}$, which indicates that the high glucose level may not be the primary reason for altering the expression and activity. The impaired secretion of other hormones, such as growth hormone, testosterone, and tetraiodothyronine, have been demonstrated in diabetics ${ }^{[44]}$, which becomes a factor inducing hepatic Cyp3a. The mechanism inducing Oatp2 expression was not reported. In the present study, we reported that DM rats showed a trend toward increasing hepatic Oatp2 levels, which was identical with the findings in type 1 diabetic rats induced by STZ ${ }^{[21]}$. We also noted that alterations of Cyp3a1, Bcrp and Mrp2 in the liver and intestines induced by diabetes were different. Our previous studies showed that alterations in activity or expressions of Cyp3a, Bcrp, Mrp2, and Oatp2 in diabetic rats were dependent on tissues and genes ${ }^{[19,21,45,46]}$. However, the real mechanisms resulting in the differential alterations were not clear and require additional investigation.

In this article, our results showed that diabetes enhanced the metabolism of simvastatin and its hydrosalt simvastatin acid in rats. The enhancement was due, in part, from up-regulated Cyp3a activity and expression as well as increased hepatic uptake.

\section{Acknowledgements}

This work was supported by National Natural Science Foundation of China (№ 81373482 and 81102503), National Basic Research Program of China (973 Program) (2011CB505300 and 2011CB505303), and the Fundamental Research Funds for the Central Universities (ZD2014YX0026 and PT2014YX0057).

\section{Author contribution}

Dan XU and Xiao-dong LIU designed the experiments and analyzed the data; Dan XU wrote the paper; Xiao-dong LIU and Li LIU revised the paper; Feng LI, Mian ZHANG, Ji ZHANG, Can LIU, Ze-yu ZHONG, Ling-ling JIA, Meng-yue HU, Da-wei WANG, and Jie WU performed the research.

\section{References}

1 Health NIO. Third Report of the National Cholesterol Education Program Expert Panel on detection, evaluation, and treatment of high blood cholesterol in adults (Adult Treatment Panel III). NIH publication 2001; 1: 3670.

2 Yusuf S. MRC/BHF Heart Protection Study of cholesterol lowering with simvastatin in 20536 high-risk individuals: a randomised placebocontrolled trial. Commentary. Lancet 2002; 360:7-22.

3 Haffner SM, Lehto S, Rönnemaa T, Pyörälä K, Laakso M. Mortality from coronary heart disease in subjects with type 2 diabetes and in nondiabetic subjects with and without prior myocardial infarction. $\mathrm{N}$ Engl J Med 1998; 339: 229-34.

4 Eliasson B, Svensson AM, Miftaraj M, Jonasson JM, Eeg-Olofsson $\mathrm{K}$, Sundell KA, et al. Clinical use and effectiveness of lipid lowering therapies in diabetes mellitus - an observational study from the Swedish national diabetes register. PLoS One 2011; 6: e18744.

5 Colhoun HM, Betteridge DJ, Durrington PN, Hitman GA, W Neil HA, Livingstone SJ, et al. Primary prevention of cardiovascular disease with atorvastatin in type 2 diabetes in the Collaborative Atorvastatin Diabetes Study (CARDS): multicentre randomised placebo-controlled trial. Lancet 2004; 364: 685-96.

6 Kearney P, Blackwell L, Collins R, Keech A, Simes J, Peto R, et al. Efficacy of cholesterol-lowering therapy in 18,686 people with diabetes in 14 randomised trials of statins: a meta-analysis. Lancet 2008; 371: 117.

7 Marot A, Morelle J, Chouinard V, Jadoul M, Lambert M, Demoulin N. Concomitant use of simvastatin and amiodarone resulting in severe rhabdomyolysis: a case report and review of the literature. Acta Clinica Belgica 2011; 66: 134-6.

8 Björnsson EJE, Kalaitzakis E. Hepatotoxicity associated with statins: reports of idiosyncratic liver in-jury post marketing. J Hepatol 2012; 
56: $374-80$

9 Colbert JD, Stone JA. Statin use and the risk of incident diabetes mellitus: a review of the literature. Can J Cardiol 2012; 28: 581-9.

10 Prueksaritanont T, Ma B, Yu N. The human hepatic metabolism of simvastatin hydroxy acid is mediated primarily by CYP3A, and not CYP2D6. Br J Clin Pharmacol 2003; 56: 120-4.

11 leiri I, Higuchi S, Sugiyama Y. Genetic polymorphisms of uptake (OATP1B1, 1B3) and efflux (MRP2, BCRP) transporters: implications for inter-individual differences in the pharmacokinetics and pharmacodynamics of statins and other clinically relevant drugs. Expert Opin Drug Metab Toxicol 2009; 2009: 703-29.

12 Noé J, Portmann R, Brun ME, Funk C. Substrate-dependent drug-drug interactions between gemfibrozil, fluvastatin and other organic aniontransporting peptide (OATP) substrates on OATP1B1, OATP2B1, and OATP1B3. Drug Metab Dispos 2007; 35: 1308-14.

13 Pasanen MK, Neuvonen M, Neuvonen PJ, Niemi M. SLC01B1 polymorphism markedly affects the pharmacokinetics of simvastatin acid. Pharmacogenet Genomics 2006; 16: 873-9.

14 Hsiang B, Zhu Y, Wang Z, Wu Y, Sasseville V, Yang WP, et al. A novel human hepatic organic anion transporting polypeptide (OATP2) Identification of a liver-specific human organic anion transporting polypeptide and identification of rat and human hydroxymethylglutarylCoA reductase inhibitor transporters. J Biol Chem 1999; 274: 371618.

15 Hagenbuch B, Meier P. The superfamily of organic anion transporting polypeptides. Biochim Biophys Acta 2003; 1609: 1-18.

16 Sotaniemi EA, Pelkonen O, Arranto AJ, Tapanainen P, Rautio A, Pasanen M. Diabetes and elimination of antipyrine in man: an analysis of 298 patients classified by type of diabetes, age, sex, duration of disease and liver involvement. Pharmacol Toxicol 2002; 90: 155-60.

17 Marques M, Coelho E, Dos Santos N, Geleilete T, Lanchote V. Dynamic and kinetic disposition of nisoldipine enantiomers in hypertensive patients presenting with type-2 diabetes mellitus. Eur J Clin Pharmacol 2002; 58: 607-14.

18 Liu H, Liu L, Li J, Mei D, Duan R, Hu N, et al. Combined contributions of impaired hepatic cyp2c11 and intestinal breast cancer resistance protein activities and expression to increased oral glibenclamide exposure in rats with streptozotocin-induced diabetes mellitus. Drug Metab Dispos 2012; 40: 1104-12.

19 Hu N, Xie S, Liu L, Wang X, Pan X, Chen G, et al. Opposite effect of diabetes mellitus induced by streptozotocin on oral and intravenous pharmacokinetics of verapamil in rats. Drug Metab Dispos 2011; 39 : 419-25.

20 Chen G, Hu N, Liu L, Xie S, Wang P, Li J, et al. Pharmacokinetics of verapamil in diabetic rats induced by combination of high-fat diet and streptozotocin injection. Xenobiotica 2011; 41: 494-500.

21 Zhang LL, Lu L, Jin S, Jing XY, Yao D, Hu N, et al. Tissue-specific alterations in expression and function of P-glycoprotein in streptozotocin-induced diabetic rats. Acta Pharmacol Sin 2011; 32: 956-66.

22 Hasegawa Y, Kishimoto S, Shibatani N, Inotsume N, Takeuchi Y, Fukushima S. The disposition of pravastatin in a rat model of streptozotocin-induced diabetes and organic anion transporting polypeptide 2 and multidrug resistance- associated protein 2 expression in the liver. Biol Pharm Bull 2010; 33: 153-6.

23 Ellis LC, Hawksworth GM, Weaver RJ. ATP-dependent transport of statins by human and rat MRP2/Mrp2. Toxicol Appl Pharmacol 2013; 269: 187-94.

24 Keskitalo JE, Pasanen MK, Neuvonen PJ, Niemi M. Different effects of the ABCG2 c.421C>A SNP on the pharmacokinetics of fluvastatin, pravastatin and simvastatin. Pharmacogenomics 2009; 10: 1617 -
24.

25 Bonora E, Targher G, Alberiche M, Bonadonna RC, Saggiani F, Zenere $\mathrm{MB}$, et al. Homeostasis model assessment closely mirrors the glucose clamp technique in the assessment of insulin sensitivity: studies in subjects with various degrees of glucose tolerance and insulin sensitivity. Diabetes Care 2000; 23: 57-63.

26 Cibickova L, Radomir H, Stanislav M, Norbert C, Helena Z, Daniel J, et al. The influence of simvastatin, atorvastatin and high-cholesterol diet on acetylcholinesterase activity, amyloid beta and cholesterol synthesis in rat brain. Steroids 2009; 74: 13-9.

27 Yu WL, Wang SJ, Han JL, Bai ZL, Sun HH, Jin R, et al. The preventive and therapeutic effects of simvastatin on osteoporotic rats. J Xi'an Jiaotong Univ (Medical Sciences) 2009; 5: 22.

28 Liu X, Chism JP, LeCluyse EL, Brouwer KR, Brouwer KL. Correlation of biliary excretion in sandwich-cultured rat hepatocytes and in vivo in rats. Drug Metab Dispos 1999; 27: 637-44.

29 Yang $\mathrm{H}$, Feng Y, Luan Y. Determination of Simvastatin in human plasma by liquid chromatography-mass spectrometry. J Chromatogr B 2003; 785: 369-75.

30 Houston JB, Kenworthy KE. In vitro-in vivo scaling of CYP kinetic data not consistent with the classical Michaelis-Menten model. Drug Metab Dispos 2000; 28: 246-54.

31 Reed M, Meszaros K, Entes L, Claypool M, Pinkett J, Gadbois T, et al. A new rat model of type 2 diabetes: the fat-fed, streptozotocin-treated rat. Metab Clin Exp 2000; 49: 1390-4.

32 Srinivasan K, Viswanad B, Asrat L, Kaul C, Ramarao P. Combination of high-fat diet-fed and low-dose streptozotocin-treated rat: a model for type 2 diabetes and pharmacological screening. Pharmacol Res 2005; 52: 313-20.

33 Maeda K, Ikeda Y, Fujita T, Yoshida K, Azuma Y, Haruyama Y, et al. Identification of the rate-determining process in the hepatic clearance of atorvastatin in a clinical cassette microdosing study. Clin Pharmacol Ther 2011; 90: 575-81.

34 Watanabe T, Kusuhara H, Maeda K, Kanamaru H, Saito Y, Hu Z, et al. Investigation of the rate-determining process in the hepatic elimination of HMG-CoA reductase inhibitors in rats and humans. Drug Metab Dispos 2010; 38: 215-22.

35 Matzke GR, Frye RF, Early JJ, Straka RJ, Carson SW. Evaluation of the influence of diabetes mellitus on antipyrine metabolism and CYP1A2 and CYP2D6 activity. Pharmacotherapy 2000; 20: 182-90.

36 Mauro VF. Clinical pharmacokinetics and practical applications of simvastatin. Clin Pharmacokinet 1993; 24: 195-202.

37 Woodcroft KJ, Hafner MS, Novak RF. Insulin signaling in the transcriptional and posttranscriptional regulation of CYP2E1 expression. Hepatology 2002; 35: 263-73.

38 Abdelmegeed MA, Carruthers NJ, Woodcroft KJ, Kim SK, Novak RF. Acetoacetate induces CYP2E1 protein and suppresses CYP2E1 mRNA in primary cultured rat hepatocytes. J Pharmacol Exp Ther 2005; 315: 203-13.

39 Yamazoe Y, Murayama N, Shimada M, Yamauchi K, Kato R. Cytochrome P450 in livers of diabetic rats: regulation by growth hormone and insulin. Arch Biochem Biophys 1989; 268: 567-75.

40 Irizar A, loannides C. Marked inhibition of hepatic cytochrome P450 activity in cholesterol-induced atherosclerosis in rabbits. Toxicology 1998; 126: 179-93.

41 Lucas D, Farez C, Bardou L, Vaisse J, Attali J, Valensi P. Cytochrome P450 2E1 activity in diabetic and obese patients as assessed by chlorzoxazone hydroxylation. Fundam Clin Pharmacol 1998; 12: 553-8.

42 Watson AM, Poloyac SM, Howard G, Blouin RA. Effect of leptin on cytochrome P-450, conjugation, and antioxidant enzymes in the 
ob/ob mouse. Drug Metab Dispos 1999; 27: 695-700.

43 Stewart CC, Strother A. Glucose consumption by rats decreases cytochrome P450 enzyme activity by altering hepatic lipids. Life Sci 1999; 64: 2163-72.

44 Thummel KE, Schenkman JB. Effects of testosterone and growth hormone treatment on hepatic microsomal P450 expression in the diabetic rat. Mol pharmaco 1990; 37: 119-29.

45 He L, Yang Y, Guo G, Yao D, Liu HH, Sheng JJ, et al. Opposite regulation of hepatic breast cancer resistance protein in type 1 and 2 diabetes mellitus. Eur J Pharmacol 2014; 724: 185-92.

46 Zhang LL, Jin S, Li J, Duan R, Liu XD. Tissue-specific damage in expression and function for breast cancer resistance protein in streptozotocin-induced diabetic rats. J China Pharm Univ 2011: 42: 544-50. 